Editorial

\title{
Challenges and Advances in Nanotoxicology
}

\author{
Robert L. Tanguay \\ Department of Environmental and Molecular Toxicology, Oregon State University, Corvallis, \\ OR 97331-4003, USA, E-Mail: robert.tanguay@oregonstate.edu; Tel.: +1-541-737-6514; \\ Fax: +1-541-737-6074
}

Received: 11 August 2014 / Accepted: 11 August 2014 / Published: 22 August 2014

This Special Issue of Nanomaterials examines the potential for engineered nanomaterials to negatively impact biological systems and highlights some advances in evaluating key areas of their hazard potential. Nanomaterial science is evolving rapidly with the generation of more complex nanostructures with exciting potential applications. Keeping modern toxicology abreast of this innovation to the point that it guides a safer nanotechnology presents an equally exciting and eminently worthwhile challenge.

In this issue, Nogueira et al. [1] provide a critical review of current in vitro methods for measurement of nanostructure cytotoxicity. Cohignac et al. [2] review current understanding of the respiratory toxicity of nanomaterials and the possible role of autophagy, and Barna et al. [3] review the potential for carbon nanomaterial involvement in human granulomatous disease culled from epidemiological data of emergency first responders. This is followed by a new report from Totsuka et al. [4] that genotoxic effects of magnetite nanoparticles in the murine lung include oxidative stress- and inflammation related-DNA adduct formations, inflammatory cell infiltration and focal granulomatous formations. O'Shaughnessy et al. [5] report on the pulmonary sub-acute toxicity of double-walled carbon nanotubes in a murine model.

In medical nano-applications, Qiao et al. [6] report a protective role for low dose graphene oxide in normal cells against oxidative damage by X-ray radiation therapy. Papageorgiou et al. [7] address concerns about the safety of $\mathrm{CoCr}$ wear particles from synthetic spinal discs and suggest that $\mathrm{CoCr}$ nanoparticles could lead to significant secondary effects, such as neural tissue inflammation. On one of the newest nano-technology fronts, Saleh et al. [8] review how nanohybrids, conjugation of metal/metal oxides with carbonaceous nanomaterials and overcoating or doping of one metal with another, present both a new set of physicochemical properties and new challenges in hazard assessment. In ecotoxicology, Hanna et al. [9] offer new evidence that marine mussels have the potential to influence the fate and transport of $\mathrm{CuO}$ engineered nanoparticles and possibly act as a significant source of $\mathrm{Cu}$ to the marine benthos. In developmental toxicology, Harper et al. [10] report that carefully synthesized, high purity, peptide-functionalized AuNPs show high biocompatibility with the 
developing zebrafish embryo. Finally, in plant toxicology, Geisler-Lee et al. [11] report that AgNPs induce abiotic stress and cause reproductive toxicity in Arabidopsis, and Begum et al. [12] report substantial differential toxicity of multi-walled carbon nanotubes toward a variety of economically important crop species.

\section{References}

1. Nogueira, D.R.; Mitjans, M.; Rolim, C.M.; Vinardell, M.P. Mechanisms Underlying Cytotoxicity Induced by Engineered Nanomaterials: A Review of In Vitro Studies. Nanomaterials 2014, 4, 454-484.

2. Cohignac, V.; Landry, M.J.; Boczkowski, J.; Lanone, S. Autophagy as a Possible Underlying Mechanism of Nanomaterial Toxicity. Nanomaterials2014, 4, 548-582.

3. Barna, B.P.; Judson, M.A.; Thomassen, M.J. Carbon Nanotubes and Chronic Granulomatous Disease. Nanomaterials 2014, 4, 508-521.

4. Totsuka, Y.; Ishino, K.; Kato, T.; Goto, S.; Tada, Y.; Nakae, D.; Watanabe, M.; Wakabayashi, K. Magnetite Nanoparticles Induce Genotoxicity in the Lungs of Mice via Inflammatory Response. Nanomaterials 2014, 4, 175-188.

5. O'Shaughnessy, P.T.; Adamcakova-Dodd, A.; Altmaier, R.; Thorne, P.S. Assessment of the Aerosol Generation and Toxicity of Carbon Nanotubes. Nanomaterials 2014, 4, 439-453.

6. Qiao, Y.; Zhang, P.; Wang, C.; Ma, L.; Su, M. Reducing X-Ray Induced Oxidative Damages in Fibroblasts with Graphene Oxide. Nanomaterials 2014, 4, 522-534.

7. Papageorgiou, I.; Abberton, T.; Fuller, M.; Tipper, J.L.; Fisher, J.; Ingham, E. Biological Effects of Clinically Relevant CoCr Nanoparticles in the Dura Mater: An Organ Culture Study. Nanomaterials 2014, 4, 485-504.

8. Saleh, N.B.; Afrooz, A.R.M.N.; Bisesi, J.H., Jr.; Aich, N.; Plazas-Tuttle, J.; Sabo-Attwood, T. Emergent Properties and Toxicological Considerations for Nanohybrid Materials in Aquatic Systems. Nanomaterials 2014, 4, 372-407.

9. Hanna, S.K.; Miller, R.J.; Lenihan, H.S. Accumulation and Toxicity of Copper Oxide Engineered Nanoparticles in a Marine Mussel. Nanomaterials 2014, 4, 535-547.

10. Harper, B.; Sinche, F.; Ho Wu, R.; Gowrishankar, M.; Marquart, G.; Mackiewicz, M.; Harper, S.L. The Impact of Surface Ligands and Synthesis Method on the Toxicity of Glutathione-Coated Gold Nanoparticles. Nanomaterials 2014, 4, 355-371.

11 Geisler-Lee, J.; Brooks, M.; Gerfen, J.R.; Wang, Q.; Fotis, C.; Sparer, A.; Ma, X.; Berg, R.H.; Geisler, M. Reproductive Toxicity and Life History Study of Silver Nanoparticle Effect, Uptake and Transport in Arabidopsis thaliana. Nanomaterials 2014, 4, 301-318.

12. Begum, P.; Ikhtiari, R.; Fugetsu, B. Potential Impact of Multi-Walled Carbon Nanotubes Exposure to the Seedling Stage of Selected Plant Species. Nanomaterials 2014, 4, 203-221.

(C) 2014 by the authors; licensee MDPI, Basel, Switzerland. This article is an open access article distributed under the terms and conditions of the Creative Commons Attribution license (http://creativecommons.org/licenses/by/3.0/). 